\title{
Trabeculectomy - Prevention and Management of Complications
}

\author{
David Cordeiro Sousa ${ }^{1,2}$ and Luís Abegão Pinto ${ }^{1,2}$ \\ 1. Department of Ophthalmology, Hospital de Santa Maria, Lisbon, Portugal; 2. Vision Sciences Study Centre, CECV, Faculty of Medicine, \\ University of Lisbon, Lisbon, Portugal
}

\section{Keywords}

Glaucoma, trabeculectomy, bleb

leak, hypotony, surgical failure

Disclosures: Dr Cordeiro Sousa and Dr Abegão Pinto have nothing to declare in relation to this article. Review process: Double-blind peer review.

Compliance with Ethics: This study involves a review of the literature and did not involve any studies with human or animal subjects performed by any of the authors.

Authorship: The named authors meet the International Committee of Medical Journal Editors (ICMJE) criteria for authorship of this manuscript, take responsibility for the integrity of the work as a whole and have given final approval to the version to be published.

Open access: This article is published under the Creative Commons Attribution Noncommercial License, which

permits any non-commercial use, distribution, adaptation and reproduction provided the original authors and source are given appropriate credit. () The Authors 2018.

Received: 20 October 2018

Accepted: 28 November 2018

Citation: European Ophthalmic Review. 2018;12(2):98-101

Corresponding Author: Luís Abegão Pinto, Department of Ophthalmology, Hospital de Santa Maria,

Av. Prof Egas Moniz, 1649-035 Lisboa, Portugal.

E: abegaopinto@gmail.com

Support: No funding was received for

the publication of this article.
Initially described by Sugar and Cairns in the early 1960s, traditional trabeculectomy was associated with a significant risk of complications, such as persistent hypotony, bleb-related problems and sight-threatening infection..$^{1,2}$ since then, and motivated by the need to reduce the number of complications and surgical failure rates, trabeculectomy technique has evolved in order to minimise associated complications, while keeping an effective intraocular pressure (IOP)-lowering effect. ${ }^{1-3}$

Modern trabeculectomy remains the gold-standard surgical option in glaucoma, consisting of a guarded sclerokeratectomy that creates a permanent fistula between the sub-Tenon's space and the anterior chamber. ${ }^{3}$ The procedure has evolved with the use of viscosurgical devices to maintain the anterior chamber formed, the placement of releasable/adjustable sutures, the application of antifibrotic agents, and bleb needling in the postoperative period. All these changes allowed for a reduced number of complications and better outcomes. ${ }^{4,5}$

Nowadays, long-term success rates approach values higher than $90 \%$, with relatively low serious complication rates. ${ }^{6-8}$ However, these improved outcomes imply a careful preoperative risk stratification, a meticulous intraoperative technique and watchful postoperative care. This review discusses some trabeculectomy-related complications and various strategies to prevent or treat these conditions.

\section{Bleb-related complications - when to return to the operating room}

The integrity of the conjunctiva filtering bleb is crucial for the success of this surgery. One of the main causes of surgical failure - bleb scarring - justified the use of antifibrotics such as mitomycin C (MMC) and 5-fluorouracil (5-FU) to improve surgical success. However, these chemoadjuvants may contribute to complications such as bleb leak, blebitis and overfiltration with hypotony. ${ }^{9}$ It is thus important to know how to deal with these complications, either with medical therapy alone or with a surgical revision.

Early bleb leak (positive Seidel test)

The most common cause of an early bleb leak is an inadequate conjunctival wound closure leading to a flat bleb, shallow anterior chamber and hypotony (Figure 1). Several studies suggest an increased incidence of postoperative leakage with fornix-based conjunctival incisions, as noted by glaucoma surgeons when shifting from a limbus-based to fornix-based technique. However, the use of the latter approach is advantageous as it is easier to perform, allows for a better exposure and sclerostomy visualisation, the area of dissected conjunctiva is smaller, it may be easier to re-intervene and, most importantly, it is associated with more posteriorly located and diffuse blebs. ${ }^{5,10}$ As a serious complication, the prevention of postoperative bleb leaks is of paramount importance, with special attention paid to intraoperative conjunctival manipulation and reproducible watertight closure techniques. 


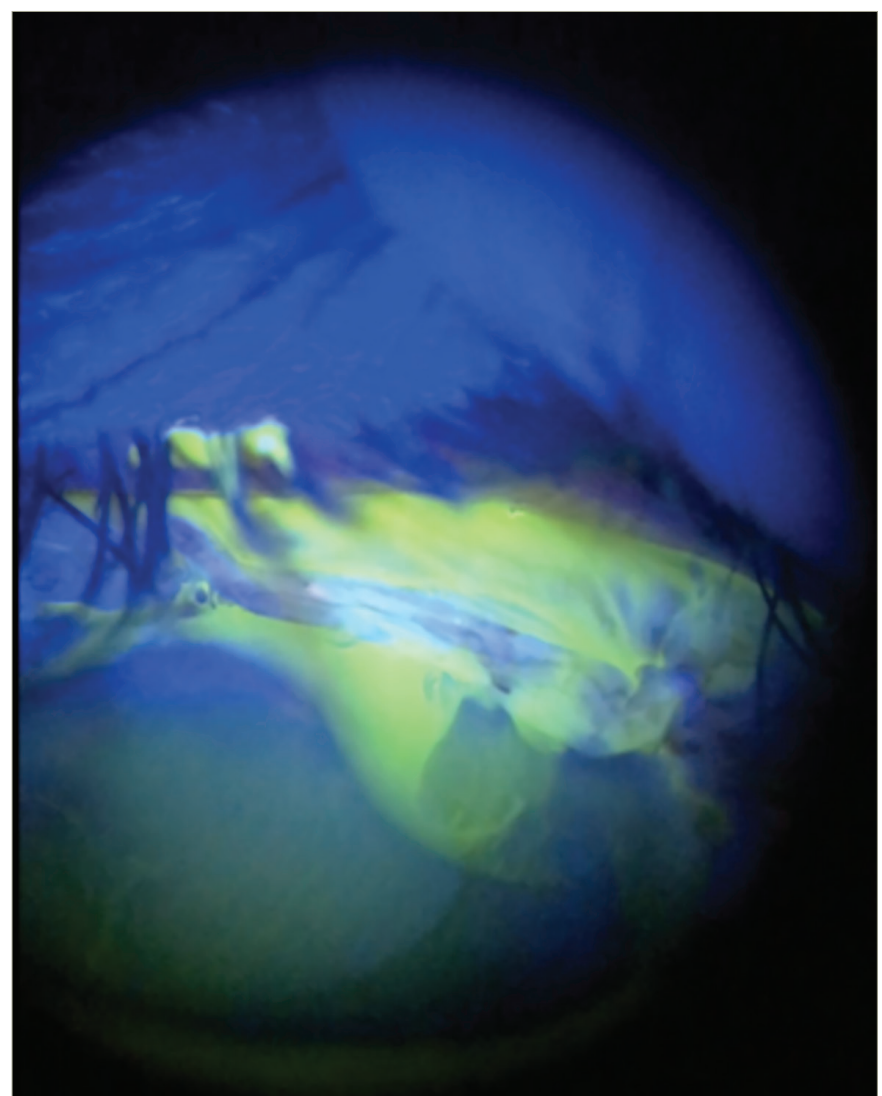

Gentle pressure can be applied to confirm or exclude an early bleb leak.

Surgical strategies to minimise the occurrence of an early bleb leak include: i) a limited conjunctival fornix-based incision; ii) careful conjunctival handling with gentle forceps to prevent buttonholes; iii) meticulous conjunctival and limbus protection during antifibrotic application; iv) precise conjunctival wound closure and v) preoperative risk stratification of patients with limited wound healing ability. ${ }^{5}$

Keep in mind, however, that even when identified postoperatively, rarely is a surgical intervention necessary to repair a bleb leak. Recent data from Alwitry et al. suggest that an early bleb leak is not necessarily a poor prognostic indicator of intermediate bleb survival. ${ }^{11}$ In their study, almost half of cases (48.1\%) were managed conservatively using a bandage contact lens, and $15 \%$ of them were managed with primary re-suturing. ${ }^{11}$ The identification of the exact site of leakage is important. A wound edge leakage after a fornix-based surgery can be managed by first ensuring that any rolled-in edge of conjunctiva on the limbal side is unfolded, and the limbal epithelium may be scraped to ensure a raw surface for better conjunctival healing. The edge should then be secured with appropriate stitches. In the case of a conjunctival buttonhole, a purse-string suture is often employed. If it is near the limbus, it can be directly sutured to the cornea or limbal sclera.? However, in many cases, for the conjunctival buttonhole or suture hole, if the location is not too far away from the limbus, a large-diameter bandage contact lens and steroid tapering may be effective.

\section{Bleb overdrainage}

The hypotony ( $<6 \mathrm{mmHg}$ ) associated with overdrainage also increased after the wide use of antifibrotic agents (especially MMC). Most cases in the early postoperative period settle without intervention. Hypotony becomes more relevant when accompanied by a shallow anterior

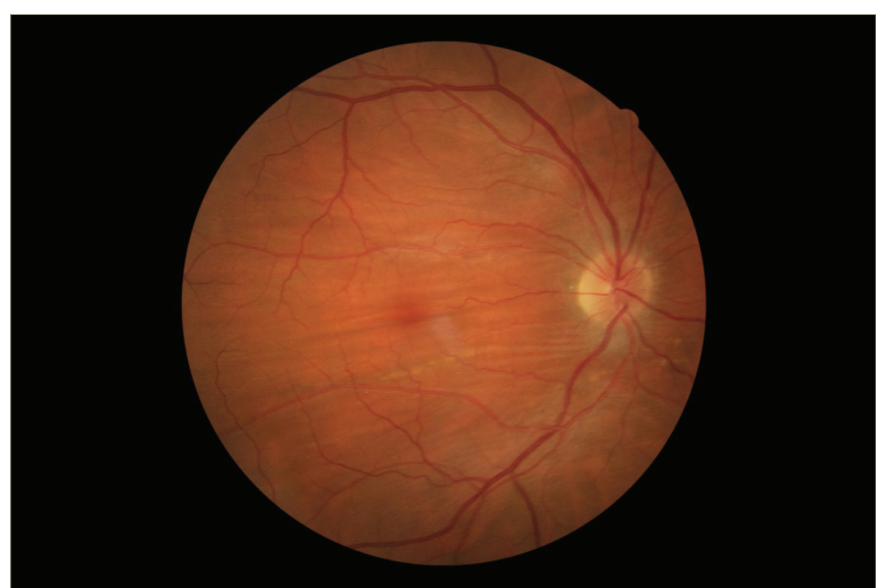

Serious hypotony may be associated in typical fundus findings, such as choriotenal folds, vascular tortuosity and blurred optic nerve head margins.

\section{Figure 3: Laser suture lysis}

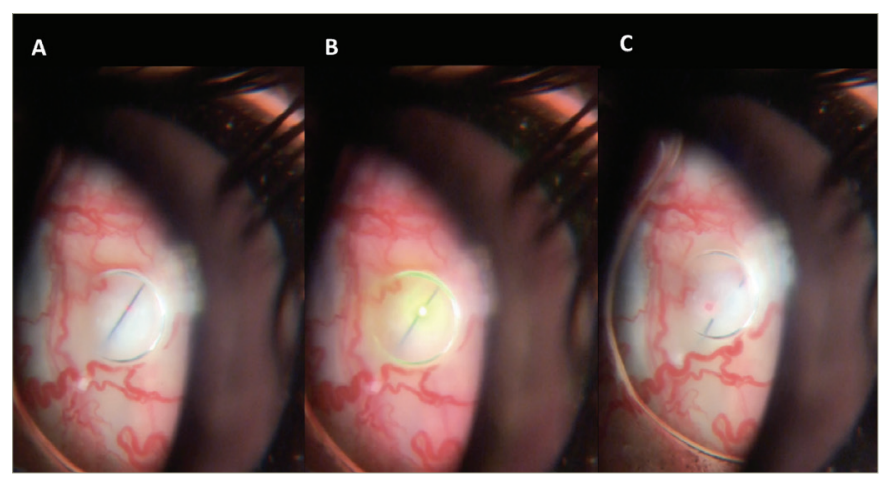

A minimally invasive procedure may be performed in the postoperative period to increase aqueous humour filtration through a subconjunctival lysis of a scleral suture. A: Identification of the suture with the Blumenthal VOLK ${ }^{\circledast}$ lens (Volk Optical, Inc., Mentor, OH, US); B: Nd:YAG Laser applied; C: suture open in the end. Suture lysis is a valid alternative to releasable sutures. It has the advantage of allowing the surgeon to perform standard, regular scleral sutures (speeding up surgery). The downside is the need for an adequate laser in the office to be able to perform the procedure. Choice between options are at the surgeon's discretion as both techniques seem to provide a similar intraocular pressure-lowering effect.

chamber, choroidal detachment or associated maculopathy (Figure 2), in which case a surgical intervention may be necessary.

Risk factors for bleb drainage include patients with myopia, collagen disease or a frailer sclera. Preventive measures include: i) a tight scleral flap closure (managed with suturolysis in the postoperative period as needed [Figure 3]), ii) avoidance of flaps that are too thin, thus not providing adequate resistance to aqueous outflow and iii) the use of adjustable sutures. ${ }^{5}$ Regarding point ii, in order to prevent hypotony related to aqueous overdrainage, small or thin scleral flaps should be avoided and, in the postoperative period, special care should be taken to not release or lyse the scleral flap suture too early - it is preferable to opt for bleb massage only according to IOP values. ${ }^{5}$

In the management of overfiltration, a risk stratification should be applied depending on the occurrence of accompanying anterior chamber shallowing, significant hypotony or choroidals. According to spaeth's classification, anterior chamber shallowing may be divided into three grades: grade I - peripheral anterior iridocorneal endothelial touch, grade II - iridocorneal endothelial touch extending from the angle to the pupil margin and grade III - a flat anterior chamber with lenticular (or vitreous) corneal endothelial touch (Figure 4). ${ }^{12}$ The absence of these signs or a 
Figure 4: Anterior segment ocular coherence tomography

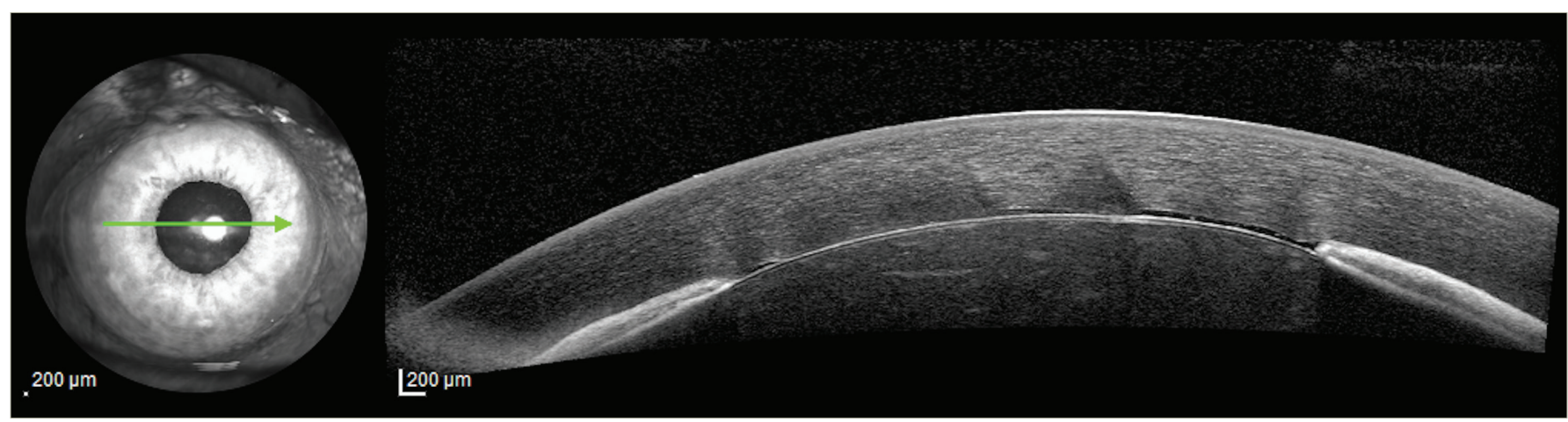

A very shallow anterior chamber revealing contact of the iris-lens diaphragm and the corneal endothelium.

Figure 5: Anterior segment colour photography denoting a dysthesic bleb

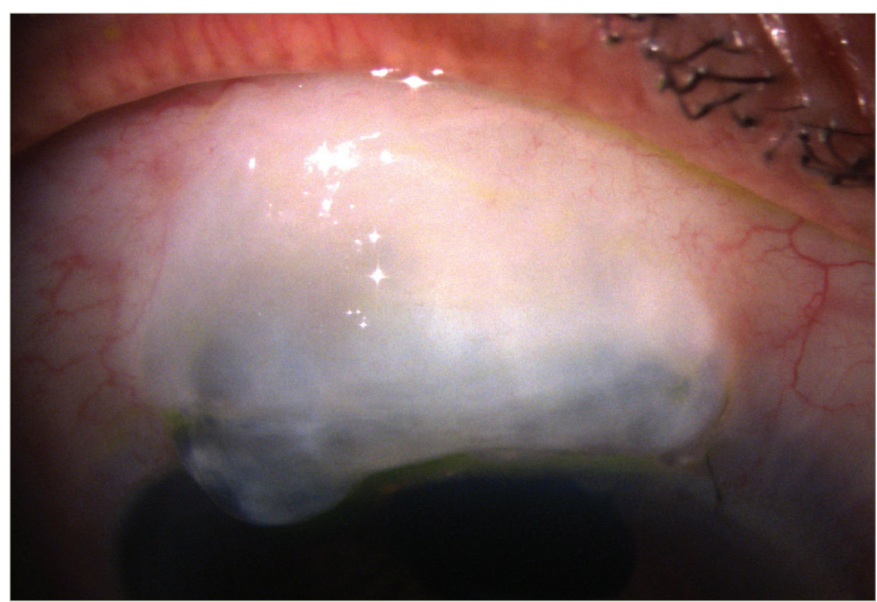

This is associated with an avascular diffuse stromal conjunctival necrosis of the conjunctiva.

grade I shallowing may be managed conservatively using cycloplegics (to pull the iris-lens diaphragm backwards and help stabilise the blood-aqueous barrier). In grades II and III the use of a viscoelastic surgical device to reform the anterior chamber may be necessary. ${ }^{9,13} \mathrm{~A}$ technique described by Palmberg consisted of compression mattress sutures for overfiltering thin-walled ischemic blebs: one or more 9-0 or 10-0 nylon mattress sutures anchored in the cornea are passed over the bleb to compress it, secured with an episcleral bite and passed back over anteriorly. ${ }^{14}$ This procedure is considered safe and effective for managing severe cases. ${ }^{14,15}$ In moderate to severe cases, dye-assisted frequency-doubled Nd:YAG laser has also been used successfully to induce conjunctival shrinkage and subsequent scarring in order to control aqueous overfiltration. Moreover, Nd:YAG-induced conjunctival vessel disruption has been suggested to help wound healing as the fibrin from the induced bleeding would increase conjunctival resistance. ${ }^{16,17}$

\section{Late bleb leaks}

Late bleb leaks occur months or years after the surgery and their occurrence is mainly related to the use of adjuvant antifibrotic agents. The leak may be focal or diffuse in nature. Preventive strategies include the avoidance of thin scleral flaps and an adequate titration of the use of antifibrotic agents, avoiding excessive exposure. ${ }^{5}$

Various therapeutic options have been described to manage late bleb leaks, namely the use of aqueous suppressants and bandage contact lenses. ${ }^{18,19}$ The former reduce the aqueous flow allowing for epithelial proliferation while bandage contact lenses promote epithelial migration and facilitate wound healing.

If a dysthesic 'sweating' bleb (associated with diffuse stromal conjunctival necrosis) occurs it is likely in need of surgical repair as it is usually both symptomatic of and a risk for infection (Figure 5). The excision of the avascular part of the bleb along with anterior mobilisation of the conjunctiva and Tenon (using relaxing posterior incisions) is advocated. ${ }^{20}$ If the conjunctiva size or status is deemed inadequate, an autologous conjunctival graft or amniotic membrane may be considered for repair as well. ${ }^{21,22}$ With a late bleb leak, surgical repair is considered more effective than conservative measures. ${ }^{20,23}$

Ultimately, it is important to emphasise the careful use of non-toothed forceps for handling the conjunctiva; blunt dissection; round-bodied suturing to help reduce conjunctival buttonholes and suture holes; and the avoidance of aggressive use of MMC or 5-FU.

\section{Chronic hypotony}

An uncommon but possible complication is the occurrence of chronic hypotony due to a cyclodialysis cleft created accidentally during the time of surgery or from minor trauma several weeks or months after trabeculectomy if the ciliary body attachment to the scleral spur is weakened. Management with cycloplegics is recommended to decrease the width of the cleft and to allow apposition of the ciliary body to the sclera. Sometimes laser photocoagulation, vitrectomy and/or cryotherapy may be needed. ${ }^{24}$

\section{Aqueous misdirection syndrome}

Although rare, aqueous misdirection syndrome may complicate simple or combined filtration procedures - most commonly in small hyperopic eyes - and is a serious and challenging situation to manage. ${ }^{25,26}$ In this condition, an aqueous misdirection occurs, with the aqueous directed posteriorly into the vitreous cavity. An anteriorly rotated ciliary body is observed, pushing against the lens equator and preventing anterior aqueous movement. Therefore, the affected patient presents with a shallow anterior chamber and raised IOP. Preventive measures include the identification of eyes with reduced axial length preoperatively, and consideration of the use of prophylactic sclerotomies in extreme cases, such as nanophthalmos. ${ }^{5,27}$

Medical management of this condition implies the initial use of atropine, topical steroids, mydriatics and topical and systemic ocular hypotensive drugs. A posterior capsulotomy/hialoidotomy should be tried and, if unsuccessful, a surgical approach with either pars 
plana vitrectomy or a zonulo-hyaloido-vitrectomy (anteriorly via a peripherical iridotomy) is needed with celerity. Another possible option would be to apply sectorial cyclodestruction to reduce ciliary body volume, thereby anatomically decreasing the overcrowding while simultaneously decreasing aqueous humour production. ${ }^{25,28}$

\section{Suprachoroidal haemorrhage}

Bleeding into the suprachoroidal space is a potentially blinding condition which may occur with any procedure in which the anterior chamber is suddenly decompressed, such as during the sclerostomy creation during the trabeculectomy. In the Tube Versus Trabeculectomy (TVT) study, this complication occurred in $3 \%$ of patients. ${ }^{29}$

A preventive approach is based on a surgical strategy that avoids sudden IOP reductions, mainly in high-risk patients such as young patients, those with eyes with very large or short axial length and patients with Sturge-Weber syndrome. During surgery, some steps are useful in this regard: pre-injection of cohesive viscoelastic into the anterior chamber, the use of preplaced scleral flap sutures and/or the use of an anterior chamber maintainer. During general anaesthesia, hyperventilation-induced vasoconstriction may also be helpful. Lastly, be aware of and consider stopping medications that predispose bleeding. 5.30

When a suprachoroidal haemorrhage occurs, it is necessary to confirm anterior chamber status and that all wounds are properly closed. Posterior segment examination determines patient management. If it is peripheral and not affecting the posterior pole, conservative measures are usually sufficient. If it is extensive, surgical drainage through a sclerotomy should be performed, before the blood clots, and using an anterior chamber maintainer. ${ }^{5,30,31}$

\section{Cataract formation}

Trabeculectomy has been shown to be associated with cataract development due to various causes, including lens-corneal touch, lens trauma, inflammation, hypotony and the use of MMC. Therefore, the avoidance of postoperative hypotony and flat anterior chamber may reduce the risk of lens opacity progression. In patients with cataract, combined cataract and glaucoma filtration surgery should be taken into consideration. ${ }^{5,32}$
In a notorious editorial, ${ }^{32}$ Jampel emphasises this by mentioning a randomised controlled trial ${ }^{33}$ in which trabeculectomy was less likely to achieve an IOP of $<17 \mathrm{mmHg}(42 \%)$ than it was to induce the need for cataract surgery within the first 3 years after trabeculectomy (50\%). In a second trial by Palanca-Capristano et al., ${ }^{34}$ a similar rate of cataract development with the need for surgery was reported. However, in this trial, a decrease in visual acuity was also reported in the group that underwent filtration surgery. In addition, both the Collaborative Initial Glaucoma Treatment Study (CIGTS) and the Advanced Glaucoma Intervention Study (AGIS) reported an increased rate of cataract development in eyes that underwent filtration surgery. Reported in CIGTS was a 5-year cumulative probability of cataract extraction of $19.0 \%$ compared with a probability of $6.5 \%$ of eyes that were initially treated with topical therapy only. In AGIS, a 78\% increase in the risk of either developing cataract or undergoing cataract surgery was reported in eyes that underwent trabeculectomy compared with those that did not..$^{35}$

When discussing the risks and benefits of glaucoma filtration surgery, these data raise the importance of discussing with the patient the relatively high likelihood of cataract development and need for surgery in the short term. Along this line, the threshold for cataract removal at the time of the trabeculectomy should be lower. As a note, when performing the phacoemulsification, the corneal incisions should be away from the bleb area to preserve its integrity.

\section{Conclusion}

This short review addressed some complications associated with glaucoma filtration surgery. An attentive patient selection, careful antimetabolite usage, meticulous surgical technique and suturing, and watchful postoperative care will make the rate of occurrence of these complications very low. However, as trabeculectomy will be in the field for many years yet, it is necessary and possible to solve the rare but potential complications effectively. In fact, as we now have 50 years of accumulated experience in this surgical technique, all possible complications have been seen, described and dealt with. Now that we are proficient in managing it, we should be cautious when considering any alternatives. Our patients will thank us for this. $\square$
1. Sugar HS. Experimental trabeculectomy in glaucoma. Am J Ophthalmol. 1961;51:623-7.

Cairns JE. Trabeculectomy: Preliminary report of a new method. Am J Ophthalmol. 1968;66:673-9.

3. Razeghinejad MR, Fudemberg SJ, Spaeth GL. The changing conceptual basis of trabeculectomy: A review of past and current surgical techniques. Surv Ophthalmol. 2012:57:1-25. Stalmans I, Gillis A, Lafaut AS, Zeyen T. Safe trabeculectomy technique: Long-term outcome. Br J Ophthalmol. 2006:90:44-7. Khaw PT, Chiang M, Shah P, et al. Enhanced trabeculectomy: Khaw PT, Chiang M, Shah P, et al. Enhanced trabeculectomy
The Moorfields Safer Surgery System. Dev Ophthalmol. 2017;59:15-35.

6. Ryan A, Saad T, Kirwan C, et al. Maintenance of perioperative antiplatelet and anticoagulant therapy for vitreoretinal surgery. Clin Experiment Ophthalmol. 2013;41:387-95.

7. Gedde SJ, Singh K, Schiffman JC, Feuer WJ. The tube versus trabeculectomy study: Interpretation of results and application to clinical practice. Curr Opin Ophthalmol. 2012;23:118-26.

8. Gedde SJ, Herndon LW, Brandt JD, et al. Postoperative complications in the tube versus trabeculectomy (TVT) study during five years of follow-up. Am J Ophthalmol. 2012;153:804-14.e1.

9. Leung DYL, Tham CCY. Management of bleb complications after Leung DYL, Tham CCY. Management of bleb complication

10. Shuster JN, Krupin T, Kolker AE, Becker B. Limbus-V fornix-based conjunctival flap in trabeculectomy: A long-term randomized study. Arch Ophthalmol. 1984;102:361-2.

1. Alwitry A, Rotchford A, Patel V, et al. Early bleb leak after trabeculectomy and prognosis for bleb failure. Eye. 2009;23:858-63.

12. Edmunds B, Thompson JR, Salmon JF, Wormald RP. The National Survey of Trabeculectomy, III: Early and late complications. Eye 2002;16:297-303.

13. Monteiro De Barros DS, Kuntz Navarro JBV, Mantravadi AV, et al. The early flat anterior chamber after trabeculectomy: A randomized, prospective study of 3 methods of management.
J Glaucoma. 2009;18:13-20.

14. Palmberg P, Zacchei AC. Compression sutures: A new treatment for leaking or painful filtering blebs. Investig Ophthalmol Vis SCi. 1996;37(3).

15. Maruyama K, Shirato S. Efficacy and safety of transconjunctival scleral flap resuturing for hypotony after glaucoma filtering surgery Graefe's Arch Clin Exp Ophthalmol. 2008:246:1751-6.

16. Kahook MY, Schuman JS, Noecker RJ. Trypan blue-assisted . Kahook MY, Schuman JS, Noecker RJ. Trypan blue-assisted
neodymium:YAG laser treatment for overfiltering bleb. J Cataract Refract Surg. 200632:1089-90.

17. Sony P, Kumar H, Pushker N. Treatment of overhanging blebs with frequency-doubled Nd:YAG laser. Ophthalmic Surg Lasers Imaging. 2004;35:429-32.

18. Blok MDW, Kok JHC, Van Mil C, et al. Use of the megasoft bandage lens for treatment of complications after trabeculectomy. Am J Ophthalmol. 1990;110:264-8.

19. Wu Z, Xu G, Weinreb RN, et al. Optic nerve head deformation in glaucoma. Ophthalmology. 2015;122:1317-29.

20. Burnstein AL, WuDunn D, Knotts SL, et al. Conjunctival advancement versus nonincisional treatment for late-onset glaucoma filtering bleb leaks. Ophthalmology. 2002;109:71-5

21. Rauscher FM, Barton K, Budenz DL, et al. Long-term outcomes of amniotic membrane transplantation for repair of leaking glaucoma filtering blebs. Am J Ophthalmol. 2007:143:1052-4.

Schnyder CC, Shaarawy T, Ravinet E, et al. Free conjunctival . Schnyder $\mathrm{CC}$, Shaarawy $\mathrm{T}$, Ravinet $\mathrm{E}$, et al. Free conjuncti
autologous graft for bleb repair and bleb reduction after trabeculectomy and nonpenetrating filtering surgery. J Glaucoma. 2002;11:10-6

23. Lin AP, Chung JE, Zhang KS, et al. Outcomes of surgical bleb revision for late-onset bleb leaks after trabeculectomy. J Glaucoma. 2013;22:21-5.

24. Ioannidis AS, Barton K. Cyclodialysis cleft: Causes and repair. Curr Opin Ophthalmol. 2010;21:150-4.

25. Lois N, Wong D, Groenewald C. New surgical approach in the management of pseudophakic malignant glaucoma. Ophthalmology. 2001;108:780-3.

26. Krix-Jachym K, Zarnowski T, Rękas M. Risk factors of malignant glaucoma occurrence after glaucoma surgery. J Ophthalmol. 2017;2017:9616738

27. Grzybowski A, Kanclerz P. Acute and chronic fluid misdirection syndrome: pathophysiology and treatment. Graefe's Arch Clin Exp Ophthalmol. 2018;256:135-54.

28. Byrnes GA, Leen MM, Wong TP, Benson WE. Vitrectomy for cary block (malignant) glaucoma. Ophthalmology. 1995;102:1308-11.

29. Gedde SJ, Feuer WJ, Shi W, et al. Treatment outcomes in the primary tube versus trabeculectomy study after 1 year of follow-up. Ophthalmology. 2018;125:650-63.

30. Jeganathan VSE, Ghosh S, Ruddle JB, et al. Risk factors for delayed suprachoroidal haemorrhage following glaucoma surgery. Br J Ophthalmol. 2008;92:1393-6.

31. Vaziri K, Schwartz SG, Kishor KS, et al. Incidence of postoperative suprachoroidal hemorrhage after glaucoma filtration surgeries in the United States. Clin Ophthalmol. 2015;9:579-84.

32. Jampel $H$. Trabeculectomy: More effective at causing cataract surgery than lowering intraocular pressure? Ophthalmology. 2009:116:173-4.

33. Wong $\mathrm{TT}$, Khaw PT, Aung T, et al. The singapore 5-Fluorouracil Trabeculectomy Study: Effects on intraocular pressure control and disease progression at 3 years. Ophthalmology. 2009;116:175-84

34. Palanca-Capistrano AM, Hall J, Cantor LB, et al. Longterm outcomes of intraoperative 5 -fluorouracil versus intraoperative mitomycin $\mathrm{C}$ in primary trabeculectomy surgery. Ophthalmology. 2009;116:185-90.

35. Panarelli JF, Banitt MR, Sidoti PA, et al. Clinical impact of 8 prospective, randomized, multicenter glaucoma trials. J Glaucoma. 2015;24:64-8. 